\title{
Desarrollo de una plataforma de evaluación de estudiantes de nuevo ingreso en educación superior
}

\section{Development of a student entrance examination platform in higher education}

\author{
MARTÍNEZ-LÓPEZ, Fernando José†*, GUTIERREZ-TORRES, Luis Germán y VEGA-CHÁVEZ, \\ Efrén
}

Instituto Tecnológico Superior del Sur de Guanajuato-Tecnológico Nacional de México

ID $1^{\mathrm{er}}$ Autor: Fernando José, Martínez-López / ORC ID: 0000-0001-9781-1800

ID $1{ }^{\text {er }}$ Coautor: Luis Germán, Gutierrez-Torres / ORC ID: 0000-0001-7912-2147

ID $2^{\text {do }}$ Coautor: Efrén, Vega-Chávez, ORC ID: 0000-0001-8918-3170

DOI: $10.35429 /$ JITC.2019.7.3.6.12

Recibido 06 de Enero, 2019; Aceptado 30 de Marzo, 2019

\begin{abstract}
Resumen
El presente artículo muestra el proceso de diseño, desarrollo y puesta en marcha de una plataforma creada con el objetivo de conformar un entorno para identificación y caracterización oportuna de conocimientos, habilidades y perfiles de los estudiantes de nuevo ingreso, mediante el tratamiento de datos y tecnologías de la información, durante el proceso de exámenes de admisión institucionales en educación superior. Para su construcción, se trabajó mediante una metodología fundamentada en las mejores prácticas de ingeniería de software, desarrollando diversos módulos que integrados logran la funcionalidad requerida para fungir como plataforma de administración y análisis de información. Los antecedentes encontrados permitieron determinar que antes del planteamiento de esta plataforma, solamente se contaba con software generalizado, adaptable con algunas dificultades, destinado a la aplicación de exámenes, distante de la visión planteada de una plataforma para exploración de datos. Este trabajo pretende brindar a la institución sede y a todas las instituciones de educación superior públicas y/o privadas, una herramienta que les permita contar con bases para la toma oportuna de decisiones y la toma de acciones pertinentes que contribuyan a la formación adecuada de profesionistas desde sus etapas iniciales, mediante una identificación temprana de habilidades y perfiles de sus aspirantes.
\end{abstract}

Evaluación, Aspirantes, Software

\begin{abstract}
This article shows the process of design, development, and implementation of a platform created to form an environment for identification and timely characterization of knowledge, skills, and profiles of new students, through the treatment of data and information technologies, during the process of institutional admission exams in higher education. For its construction, work has been done through a methodology based on best software engineering practices, developing various modules that integrated achieve the functionality required to function as information management and analysis platform. The antecedents found allowed to determine that before the approach of this platform, there was only generalized software, adaptable with some difficulties, destined to the application of exams, distant from the raised vision of a platform for data exploration. This work aims to provide the host institution and all public and / or private higher education institutions with a tool that allows them to have bases for timely decision making and the taking of relevant actions that contribute to the proper training of professionals from its initial stages, through an early identification of skills and profiles of its applicants.
\end{abstract}

Citación: MARTÍNEZ-LÓPEZ, Fernando José, GUTIERREZ-TORRES, Luis Germán y VEGA-CHÁVEZ, Efrén. Desarrollo de una plataforma de evaluación de estudiantes de nuevo ingreso en educación superior. Revista de Tecnologías de la Información y Comunicaciones. 2019. 3-7: 6-12

$\uparrow$ Investigador contribuyendo como primer autor. 


\section{Introducción}

En la actualidad, las tecnologías de la información (TI) son muy relevantes en el entorno académico. Instituciones de educación alrededor del mundo incorporan cada vez más TI a sus procesos en el intento de mejorar su calidad de servicio y garantizar una enseñanzaaprendizaje de calidad. Por tanto, en la educación existe cada vez más presencia de las TI en todos los niveles, buscando brindar al estudiante herramientas que le permitan vivir un proceso educativo con mejor satisfacción. Desde hace décadas, la aplicación de exámenes de admisión en instituciones de educación superior es un hecho común, sin embargo son aún pocas las instituciones que llegan a utilizar plataformas de TI para facilitar el proceso de aplicación de estos instrumentos y menos son aquellas que hacen uso de los datos recabados para realizar una adecuada explotación de los mismos con el objetivo de brindar un mejor servicio a sus estudiantes.

\section{Actualmente las evaluaciones} automatizadas tienen un papel fundamental en la educación moderna, ya que aceleran y mejoran el proceso de aplicación, no obstante, si la herramienta de TI que permite realizar la aplicación de la evaluación no permite realizar una adecuada explotación de datos para la toma de decisiones, esta herramienta actualmente podría considerarse incompleta. Mediante el planteamiento de este proyecto, el cuerpo académico de "Tecnologías de la Información", con registro PRODEP ITESSGTO-CA-2, de la institución sede: Instituto Tecnológico Superior del Sur de Guanajuato (ITSUR) del Tecnológico Nacional de México, proyectó brindar a las instituciones de educación superior no solamente un software para la aplicación de exámenes de ingreso, sino una herramienta para la toma de decisiones, que permita determinar acciones pertinentes que contribuyan a la formación adecuada de estudiantes mediante una identificación oportuna de sus habilidades y perfiles, la plataforma denominada: Sistema Integral de Evaluación al Ingreso (SIEVALI).

\section{Antecedentes}

El uso de herramientas de TI se ha convertido al día de hoy en una tarea cotidiana y de gran valor, pues estas permiten incrementar la facilidad y agilidad con la que se desarrollan diversas actividades.
(Centeno Brambila \& Lira Obando, 2014) señalan que una gran cantidad de instituciones educativas hoy en día utilizan herramientas disponibles en Internet para mejorar sus procesos de enseñanza-aprendizaje. El uso de pruebas estandarizadas en entornos educativos es frecuente, existen diversos tipos de pruebas de acuerdo a (Jornet Meliá \& Suárez Rodríguez, 1996), como lo son: test de dominio, test de objetivos, test de competencias, test de certificación, test de criterio, test de clase o de uso en el aula, etcétera.

Existen hoy en día diversas plataformas en línea que facilitan llevar las pruebas estandarizadas a las TI y le ofrecen al usuario la oportunidad de reducir drásticamente el tiempo necesario para la revisión de las evaluaciones, además de que algunas ofrecen herramientas de estadística general. Algunas de las plataformas más conocidas son: ExamTime, Encuestafacil, Google Forms, Flubaroo y ClassMaker. Cualquiera de estas herramientas podría ser utilizada para llevar a cabo las evaluaciones de un proceso de admisión en instituciones educativas, pero cada una de ellas tiene características particulares que deben ser adaptadas para garantizar que el entorno sea el adecuado para su aplicación y que los resultados obtenidos puedan ser explotados.

Además de las plataformas que existen en Internet, también se han desarrollado plataformas que cubren algunas de las necesidades específicas de un centro educativo en particular, tal es el caso de Computer-Based Test (CBT). El software CBT, acorde a (Fagbola Temitayo, Adigun Adebisi, \& Oke Alice, 2013) está diseñado para facilitar el proceso de evaluación y además generar reportes de los resultados, no obstante, no es una plataforma accesible para cualquier institución.

En algunos centros educativos, se ha desarrollado un software específico para el proceso de admisión a la institución. (Mamani Garavito, 2013), menciona el desarrollo de un software para realizar con eficiencia la administración y gestión de la base de datos para el proceso de admisión del Instituto de Educación Superior Tecnológico Privado del Altiplano de Puno, en Perú, pero de la misma forma, es un software no accesible para cualquier centro de educación. 
En la misma institución sede, el ITSUR, se han realizado pruebas con algunas de estas plataformas para la aplicación de exámenes de ingreso, incluso se ha desarrollado un software base para validar la posibilidad del desarrollo de una plataforma integrable con el software de Sistema Integral Control Escolar (SICE), el cual, como se menciona en (Vega-Olvera, VegaFlores, \& Morales-Orozco, 2017), fue desarrollado por docentes y alumnos de la misma institución y actualmente es la plataforma de facto para llevar a cabo las actividades de control escolar.

Además, el SICE expone servicios web que proporcionan datos académicos sobre alumnos y docentes, teniendo con esto una ventaja importante mediante la interoperabilidad de servicios web, debido a que hace posible el desarrollo de aplicaciones en distintas plataformas y lenguajes de programación que permitan fortalecer los diversos procesos alrededor de las gestiones académicas de los aspirantes y estudiantes de esta institución.

La interoperabilidad del SICE, permite que otros sistemas aprovechen la infraestructura ya existente, de manera que el ITSUR cuente con una plataforma integrada donde varios sistemas coexisten. En la actualidad se encuentran los siguientes: Psicotest, del que se presentan resultados en (Morales-Orozco, GutiérrezTorres, \& Vega-Olvera, 2016), SICEDroid y el Sistema de Evaluación Docente, ambos elaborados para la institución de manera que forman un antecedente base en el desarrollo de este tipo de plataformas.

\section{Descripción del método}

Cómo ya es conocido, el desarrollo de nuevas tecnologías de la información no es un proceso simple, sobre todo las plataformas de software, pues requieren la integración de toda una colección de buenas prácticas de ingeniería, como lo sustentan (Jacobson, Ng, E. McMahon, Spence, \& Lidman, 2012).
La dinámica de trabajo que ha seguido el cuerpo académico ITESSGTO-CA-2, respecto al desarrollo de proyectos tecnológicos referentes a software, ha sido la de una metodología de trabajo creada a partir de la destilación de buenas prácticas de diversos marcos de trabajo y que fue producto de trabajos realizados con el objetivo de alcanzar la acreditación en un nivel 3 de madurez en el ITSUR, correspondiente a la Integración de Modelos de Madurez de Capacidades o Capability Maturity Model Integration (CMMI, por sus siglas en inglés), documentado por (Carnegie Mellon Software Engineering Institute, 2006) y por (Chrissis, Konrad, \& Shrum, 2011), y que fue satisfactoriamente alcanzada como se describe en (GutiérrezTorres, Morales-Orozco, \& Alcantar-Ortíz, 2017).

La metodología fue fundamentada en sus orígenes en el Rational Unified Procces (RUP) de (Jacobson, Booch, \& James, The Unified Software Development Process, 1999) y fortalecida posteriormente por el Personal Software Process y Team Software Process descritos en (Pomeroy-Huff, Cannon, Chick, Mullaney, \& Nichols, 2009). La metodología además integra conocimientos ágiles dirigidas al cumplimiento del manifiesto planteado por (Kent, y otros, 2001), incorporando las buenas prácticas del marco de trabajo SCRUM definido originalmente por (Schwaber \& Sutherland, 2013).

De manera general, la metodología consistió en el desarrollo de diferentes actividades, comenzando por la realización del proceso de Ingeniería de Requisitos, como se plantea por el (International Institute of Business Analysis, 2006), llevado a cabo mediante diversos talleres de requisitos, en los que de manera directa, cara a cara, en colaboración con un grupo institucional dedicado a la aplicación de exámenes de evaluación al ingreso, se comenzó a identificar las características que serían requeridas por la plataforma para cubrir los procesos elementales de la aplicación del instrumento de evaluación a un grupo de aspirantes de nuevo ingreso, procurando en todo momento realizar la validación de cada característica detectada, con la finalidad de minimizar problemas futuros debido a especificaciones incorrectas. 
El resultado de esta actividad fue la identificación inicial de 4 épicas (Configuración de Exámenes, Aplicación de Exámenes, Reportes Administrativos y Acceso Seguro al Sistema), contemplando un total de 21 características distribuidas en estas a manera de historias de usuario. Posteriormente, acorde a la metodología, se realizó el uso del método de Análisis de Arquitectura de Software Basado en Escenarios especificado por (Kazman, Abowd, Bass, \& Clements, 1996) y el Análisis de tradeoff de la Arquitectura especificado por (Kazman, Klein, \& Clements, ATAM: Method for Architecture Evaluation, 2000), para lograr construir la definición del estilo arquitectónico y la correspondiente descomposición modular del sistema en desarrollo.

El resultado de esta actividad fue la identificación de los niveles y estrategias para el cumplimiento de los distintos atributos de calidad, así como la descomposición modular de forma tal que pudiese determinarse el estilo arquitectónico a utilizar, siendo por las características determinadas una descomposición en: Datos Centralizados y Componentes Independientes distribuidos acorde a los patrones arquitectónico en capas (Layers) para el backend y ModelViewControler para el frontend. Finalmente, debido a las características tales de una plataforma web, se consideraron los patrones de diseño: Client-Server, Publisher-Subscriber, Master-Slave y Proxy, bajo los cuales fueron realizados los diversos componentes del sistema. La descomposición modular se presenta en la continuación.

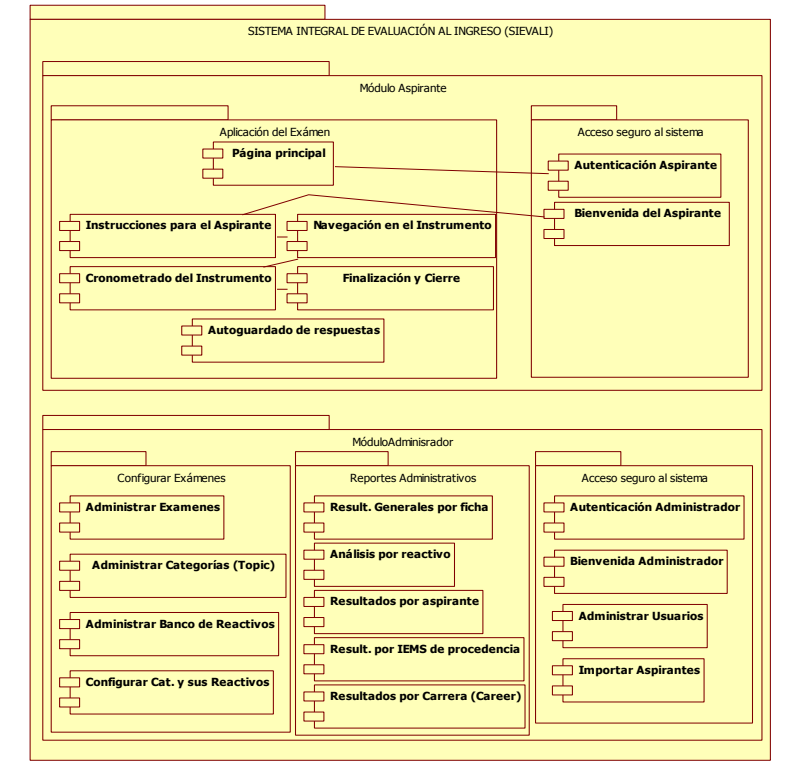

Figura 1 Descomposición modular SIEVALI

Fuente: Elaboración Propia

ISSN 2531-2200

ECORFAN® Todos los derechos reservados
Una vez establecido un marco arquitectónico, se procedió a la elección de tecnologías para la construcción de la plataforma, siendo que por la experiencia de los integrantes del cuerpo académico, fueron elegidas para el desarrollo del Front-End, diversas tecnologías web abiertas (HTML5, CSS3, Javascript, Bootstrap, JQuery, entre otros frameworks) y para el Back-End, las tecnologías correspondientes al .Net Framework correspondiente a Microsoft (2018), mediante programación en el lenguaje C\# y conectividad tradicional a base de datos relacional MySQL, utilizando un entorno de desarrollo gratuito proporcionado y licenciado por Microsoft denominado Visual Studio edición Community, el cual brinda a equipos de trabajo pequeños, las herramientas para el desarrollo rápido de aplicaciones.

El trabajo realizado fue colocado para su control de versiones en repositorios de la misma plataforma Microsoft mediante GitHub la cual es una plataforma que permite hospedar y realizar el seguimiento a cambios en el código fuente (GitHub Inc. Microsoft, 2019), además de vincular la administración de proyectos mediante kanban, con herramientas como ZenHub e integrar los cambios junto a otros desarrolladores de manera asíncrona.

Posteriormente, durante la fase de desarrollo, fueron llevados a cabo 6 sprints, con duración de 14 días, en los que se fueron realizando las distintas características planteadas en las historias de usuario detectadas. En una primera etapa, se llevó a cabo la implementación de la plataforma de aplicación de examen de admisión de manera que esta permitiera la visualización-navegación en el instrumento de evaluación (examen), así como el diseño del esquema de datos que soportaría la futura creación, modificación y eliminación de evaluaciones, reactivos, categorías, respuestas, aspirantes y sus resultados, así como la futura implementación de módulos de análisis y reporteo administrativos a partir de los datos capturados mediante la plataforma.

Este primer esfuerzo, permito elaborar el diseño del esquema relacional que puede apreciarse en la figura 2 así como la creación del arquetipo de solución en Visual Studio basado en una arquitectura en capas. 
Además del diseño inicial de las pantallas de FrontEnd correspondientes a la visualización del instrumento de evaluación que puede apreciarse en la figura 3 considerando algunos aspectos que procurarían obtener un nivel adecuado de experiencia del usuario con la plataforma.

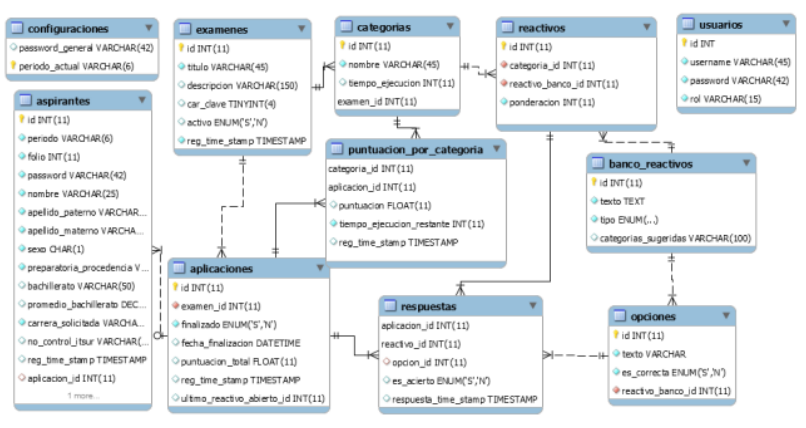

Figura 2 Esquema relacional inicial SIEVALI Fuente: Elaboración Propia

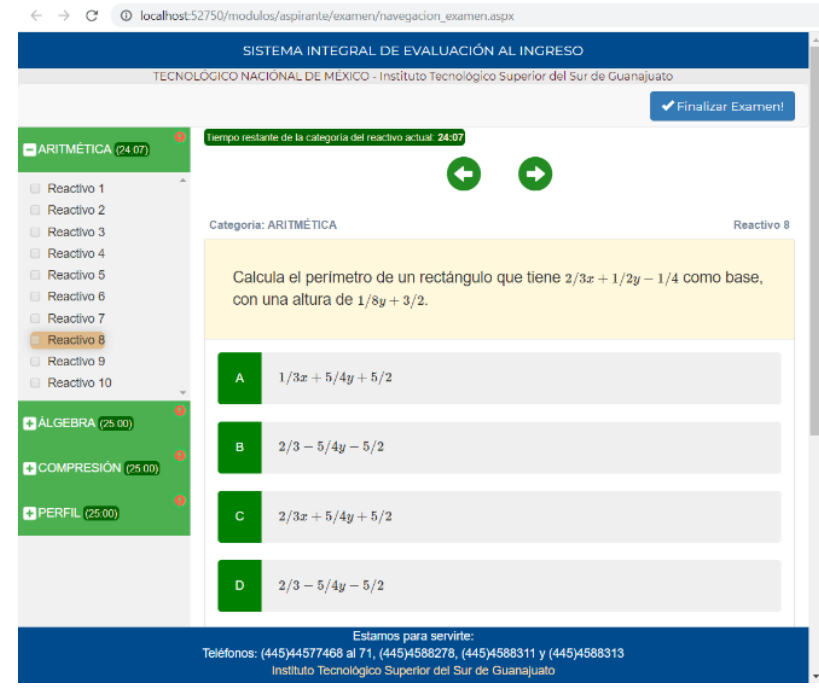

Figura 3 Diseño inicial de pantalla de visualización del instrumento de evaluación

Fuente: Elaboración Propia

Mientras transcurría el desarrollo de esta primera etapa, el grupo institucional dedicado a la aplicación de exámenes de evaluación al ingreso profesores, trabajó de manera paralela en el diseño de un banco de reactivos validados y categorizados, que integrados a la plataforma fortalecerían su valor de uso hacia futuras intensiones de uso de la misma institución u otras interesadas. Durante esta fase se llevó a cabo la compilación y validación de reactivos en las diversas categorías que fueron establecidas para el diseño de la evaluación institucional: Aritmética, Álgebra, Comprensión Lectora y Perfil.
Cada uno de estos reactivos fueron diseñados para fungir como base para que el departamento administrador de la evaluación al ingreso cuente con la posibilidad de elegir los que considere más adecuados de acuerdo al perfil de las carreras de las que disponga la institución.

Tras el logro de las Épicas esenciales para la aplicación de una evaluación en ambiente de producción, y la incorporación de características de robustez, que permitían soportar las caídas de energía o la pérdida de conexión durante la ejecución de la evaluación, considerando una versión estable de la plataforma, esta fue desplegada en ambiente de pre-producción, con la finalidad de llevar a cabo una prueba piloto. Para esto se debió realizar las configuraciones necesarias de la infraestructura proporcionada por el ITSUR, para el despliegue de la plataforma, incluyendo la preparación apropiada del servidor Web y la configuración de los servicios necesarios dentro del sistema operativo para su despliegue a través de la intranet.

Una vez desplegada la plataforma, esta pudo ser piloteada para comprobar su adecuado funcionamiento en ambiente semi controlado. Para esto se recibió el apoyo de varios grupos de estudiantes de la carrera de Ingeniería en Sistemas Computacionales del ITSUR, como se aprecia en la figura 4 quienes con su perspicacia colaboraron en identificar oportunidades de mejora y defectos que fueron incorporados al Backlog del proyecto y posteriormente priorizados para su atención oportuna.

Además se logró verificar, durante esta prueba, que las capacidades de respuesta, uso de memoria, y carga de sistema, tanto del servidor Web, como del servidor de base de datos, se mantuvieron en niveles muy satisfactorios aún con la carga de usuarios simultáneos a la que fue sometida la plataforma. 


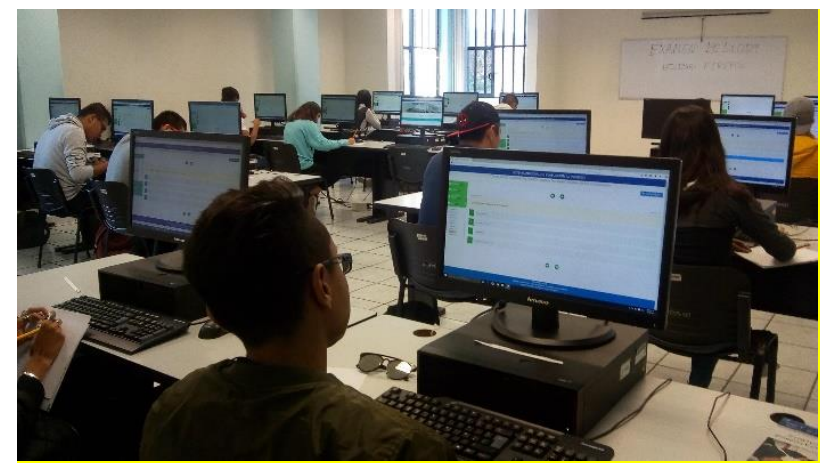

Figura 4 Ejecución de una prueba mediante la plataforma Fuente: Elaboración Propia

Posteriormente, se comenzó con un periodo de preparación para atender las oportunidades de mejora y defectos priorizados y para desarrollar algunas de las características para el procesamiento de información y módulos de reporteo.

Esto fue llevado a cabo mediante el lanzamiento de varios sprint en los que fueron realizadas las características restantes; las cuales una vez concluidas permitieron alcanzar un suficiente nivel de desarrollo para promover el uso de la plataforma en el proceso de admisión de junio 2019 del ITSUR, el cual fue llevado a cabo con éxito.

\section{Resultados}

Se puede considerar que el resultado principal obtenido tras realizar las actividades anteriormente descritas, fue en síntesis: la creación de las bases sólidas de un entorno para la evaluación automatizada de conocimientos en exámenes de admisión institucionales, una primer versión estable de la plataforma, validada tanto por prueba piloto como por prueba en ambiente real durante el proceso de admisión de junio 2019 del ITSUR.

Los resultados de la evaluación aplicada en el proceso de admisión del ITSUR serán revisados posteriormente con mayor detalle en un posterior artículo, cuyo enfoque será la valoración misma de los resultados por los departamentos de Desarrollo Académico y jefaturas relacionadas, quienes son aquellos quienes pueden aprobar el uso de la información en la maximización de la identificación de habilidades y detección de perfiles de los aspirantes, mediante el tratamiento de datos que facilita la plataforma.
Por el momento, solamente se puede hacer mención de que los resultados fueron satisfactorios y pudieron contrastarse favorablemente con los resultados de la evaluación paralela realizada durante el proceso de admisión junio 2019 del ITSUR, mediante la aplicación de un instrumento del Centro Nacional de Evaluación para la Educación Superior (Ceneval). De esto se comentará en un artículo adicional.

\section{Agradecimiento}

De manera especial se agradece el apoyo otorgado por parte del Tecnológico Nacional de México el cual por medio del financiamiento otorgado por la convocatoria 2019 de Apoyo a la Investigación Científica y Tecnológica en programas educativos de los Institutos Tecnológicos Federales, Descentralizados y Centros, facilitó los recursos necesarios para el desarrollo adecuado de este proyecto.

\section{Conclusiones}

Es posible concluir que tras la experiencia de desarrollo, prueba y despliegue de la plataforma, los resultados alcanzados al momento fueron satisfactorios, no obstante, aún quedan pasos importantes por lograr, pues se están realizando esfuerzos por preparar el despliegue oficial de la plataforma a través de Internet, para permitir el acceso simultaneo a diversas instituciones de educación superior, brindando la plataforma misma como un servicio según sea solicitado.

Es pues este artículo una manera de difundir la plataforma a otras instituciones que pudiesen estar interesadas, pues será de gran placer para el cuerpo académico conocer las opiniones y retroalimentación de otras instituciones para realizar futuras mejoras.

\section{Referencias}

Carnegie Mellon Software Engineering Institute. (2006). CMMI for Development $v$ 1.2. Pittsburgh,PA: Carnegie Mellon University.

Centeno Brambila, D. A., \& Lira Obando, A. (2014). Sistema de evaluaciones en línea como herramienta para los niveles de educación media superior. Revista Iberoamericana para la Investigación y el Desarrollo Educativo, 1-25. 
Chrissis, M. B., Konrad, M. D., \& Shrum, S. (2011). CMMI: guidelines for process integration and product improvement. AddisonWesley Professional.

Fagbola Temitayo, M., Adigun Adebisi, A., \& Oke Alice, O. (2013). Computer-based test (CBT) system for university academic enterprise examination. International Journal of Scientific \& Technology Research, 336-342.

GitHub Inc. Microsoft. (agosto de 2019). GitHub. Obtenido de github.com: https://github.com/

Gutiérrez-Torres, L. G., Morales-Orozco, D., \& Alcantar-Ortíz, P. (Diciembre de 2017). Célula de software y su relación con el desarrollo docente. Revista de Educación Superior, Vol.1(No.2), 27-31. Obtenido de http://www.ecorfan.org/republicofperu/research _journals/Revista_de_Educacion_Superior/vol1 num2/Revista_de_Educaci\%C3\%B3n_Superior _V1_N2_4.pdf

International Institute of Business Analysis. (2006). A Guide to the Business Analysis Body of Knowledge. International Institute of Business Analysis. Obtenido de http://www.iiba.org/babok-guide.aspx

Jacobson, I., Booch, G., \& James, R. (1999). The Unified Software Development Process. Boston, MA, USA: Addison-Wesley Longman Publishing Co., Inc.

Jacobson, I., Ng, P.-W., E. McMahon, P., Spence, I., \& Lidman, S. (2012). The Essence of Software Engineering: A thinking framework in the form of an actionable kernel. ACMQUEUE. Obtenido de http://delivery.acm.org/10.1145/2390000/23896 16/p40-

jacobson.pdf?ip=187.217.232.6\&id $=2389616 \&$ acc $=O P E N \& k e y=4 D 4702 B 0 C 3 E 38 B 35 \% 2 E 4 D$ 4702B0C3E38B35\%2E4D4702B0C3E38B35\% 2E6D218144511F3437\&_acm_ $=153296965$ 5_10d62d323ae9c00243220b7a52b6dc24

Jornet Meliá, J., \& Suárez Rodríguez, J. (1996). Pruebas Estandarizadas y Evaluación del Rendimiento: Usos y Características. Revista de Investigación Educativa.
Kazman, R., Abowd, G., Bass, L., \& Clements, P. (November de 1996). Scenario-Based Analysis of Software Architecture. IEEE Software, 1-14. Obtenido de http://resources.sei.cmu.edu/asset_files/WhiteP aper/1996_019_001_29912.pdf

Kazman, R., Klein, M., \& Clements, P. (2000). ATAM: Method for Architecture Evaluation. Pittsburgh, PA: Carnegie Mellon Software Engineering Institute.

Kent, B., Mike, B., van Bennekum, A., Cockburn, A., Cunningham, W., Fowler, M., . . - Thomas, D. (2001). Manifiesto por el Desarrollo Ágil de Software. Obtenido de http://agilemanifesto.org:

http://agilemanifesto.org/iso/es/manifesto.html

Mamani Garavito, R. V. (2013). Tesis: Software para el proceso de admisión y la eficiencia de la evaluación en el Instituto de Educación Superior, Tecnológico Privado Del Altiplano Puno. Perú: Universidad Nacional del Altiplano - Puno.

Morales-Orozco, D., Gutiérrez-Torres, L. G., \& Vega-Olvera, G. I. (2016). Test psicométricos: desarrollo de un software aplicando un modelo de calidad internacional. . Revista Administracion y Finanzas, 47-51.

Pomeroy-Huff, M., Cannon, R., Chick, T. A., Mullaney, J., \& Nichols, W. (2009). The Personal Software Process (PSP) Body of Knowledge, Version 2.0. (P. S. Pittsburgh, Ed.)

Schwaber, K., \& Sutherland, J. (Julio de 2013). La Guía de Scrum: La Guía Definitiva de Scrum: Las Reglas del Juego. Obtenido de WwW.scrumguides.org:

https://www.scrumguides.org/docs/scrumguide/ v1/scrum-guide-es.pdf

Vega-Olvera, G. I., Vega-Flores, P., \& MoralesOrozco, D. (2017). Servicios escolares Android: caso de estudio en la enseñanza de la programación para tecnologías móviles. Revista Electrónica ANFEI Digital, 1-8. 